\title{
Funding Mechanism Code
}

National Cancer Institute

\section{Source}

National Cancer Institute. Funding Mechanism Code. NCI Thesaurus. Code C93571.

A coded value specifying the unique identifier for the type of award an ag ency makes to support a program and within which it incorporates the terms and conditions for expending the funds and performing the work. 